International Journal of Dentistry and Oral Science (IJDOS)

ISSN: 2377-8075

\title{
Light Emitting Diode in Comparison to Halogen Curing Technology: Microshear Bond Strength of Dental Composite Resin Restorative Material
}

Hammouda $\mathrm{IM}^{1,2^{*}}$, Beyari $\mathrm{MM}^{3}$

' Professor of Dental Biomaterials, Department of Dental Biomaterials, Faculty of Dentistry, Mansoura University, Egypt.

${ }^{2}$ Head of Conservative Dentistry Department, Faculty of Dentistry, Umm Al Qura University, Makkah, KSA.

${ }^{3}$ Associate Professor of Prosthetic \& Oral Medicine, Dean, Faculty of Dentistry, Umm Al-Qura University, Makkah, KSA

\begin{abstract}
The aim of this study was to compare the micro-shear bond strength of composite resin cured with halogen visible light and blue light emitting diode curing units. Flat enamel surfaces of 50 segments of teeth were prepared. 50 cylindrical composite resin specimens were bonded to the prepared teeth segments. Specimens were divided into 5 groups, three groups were cured for 10, 20 and 40 seconds using blue light emitting diode and 2 groups were cured for 20 and 40 seconds using halogen light. Specimens were submitted to micro-shear bond test at $1 \mathrm{~mm} / \mathrm{min}$ cross-head speed. Mean micro-shear bond strengths were analyzed by analysis of variance (One-way ANOVA) and Tukey's test at $\mathrm{P}<0.05$. At 40 seconds curing, the halogen light produced significantly higher bond strength than that of blue light emitting diode. No significant difference was found between both lights at 20 seconds. There were no significant differences in bond strength ratios between 10 and 20 seconds blue light emitting diode, and 20 seconds halogen light. At 40 seconds, the blue light emitting diode produced significantly higher bond strength than that of blue light emitting diode at10 and 20 seconds, and halogen light at 20 seconds. It was concluded that despite the higher micro-shear bond strength of halogen light at 40 seconds, blue light emitting diode curing unit provided sufficient output to exceed minimum requirements in terms of composites' micro-shear bond strength according to ISO 11405, 1994. During incremental application of composite resin, 10 seconds blue light emitting diode curing time is sufficient to produce acceptable micro-shear bond strength values.
\end{abstract}

Keywords: Micro-Shear Bond Strength; Enamel; Halogen Light-Curing; LED Light-Curing.

\section{*Corresponding Author:}

Ibrahim M. Hammouda,

Head of Conservative Dentistry Department, Faculty of Dentistry, Umm Al Qura University, Makkah, KSA.

Tel: +966542812148

Fax: 0020502260173

E-mail: imh100@hotmail.com

Received: November 16, 2014

Accepted: January 05, 2015

Published: January 08, 2015

Citation: Hammouda IM, Beyari MM (2015) Light Emitting Diode in Comparison to Halogen Curing Technology: Microshear Bond Strength of Dental Composite Resin Restorative Material. Int J Dentistry Oral Sci. 2(1), 29-34. doi: http://dx.doi.org/10.19070/2377-8075-150007

Copyright: Hammouda IM $^{\circ}$ 2015. This is an open-access article distributed under the terms of the Creative Commons Attribution License, which permits unrestricted use, distribution and reproduction in any medium, provided the original author and source are credited.

\section{Introduction}

In the last few years, curing light technology has advanced with the introductions of high intensity halogen lights, light emitting diode lights(LED) and plasma arc lights for resin composite polymerization [1-3]. Despite their popularity, halogen technology light curing units (LCUs) have several drawbacks. For example, halogen bulbs have a limited effective lifetime of approximately 40-100 h [4,5]. In addition, the halogen LCU's bulb, reflector and filter degrade over time due to the high operating temperatures and the large quantity of heat which is produced during the duty cycles. This result in a reduction of the halogen LCU's curing effectiveness over time [4].

The argon laser curing provided better microshear bond strength than that of the halogen lights [1]. On the other hand, although the light source presented no significant influence, the use of high-intensity units is still recommended to ensure effectiveness of polymerization of the bonding resin [6]. Polymerization with both halogen and LED resulted in shear bond strength values which were above the clinically acceptable range given by Reynolds. The LED light curing units produced comparable shear bond strength to that of halogen curing units [7].

To overcome the problems inherent to halogen LCUs, solid state light emitting diode (LED) technology has been proposed for curing light activated dental materials [8]. The spectral output of gallium nitride blue LEDs falls conveniently within the absorption spectrum of camphorquinone photoinitiator (400-500 nm) present in light activated dental materials, so that no filters are required in LED LCUs. Furthermore, LEDs have an expected lifetime of several thousand hours without significant degradation of light flux over time [9]. The development of $470 \mathrm{~nm}$ wavelength LED-curing units offers an alternative to conventional halogen curing units. A number of studies have addressed the application of blue LED technology to curing of dental materials by measuring the depths of cure or by examining the irradiances of the units $[8,10]$. 
The LED-LCUs had an energy-efficient spectral output for conventional composite curing but had a lower irradiance compared with the quartz-tungsten-halogen light-curing unit (QTH-LCU, leading to reduced performance in depths of cure [11]. Curing light with an intensity of 300 milliwatts per square centimeter will effectively cure most composite shades in the 400-nanometer to 515-nm wavelength bandwidth at the curing-light tip within the manufacturers' recommended times [3,12]. The light output of commercially available diodes for resin-based composite polymerization still requires improvement and additional studies are necessary to rival the adequacy of cure of halogen-based LCUs [13].

Tensile and shear bond tests have long been the most common laboratory tests to evaluate adhesive strength of bonding systems to the tooth substrate. Unfortunately, studies have shown that tensile and shear testing is significantly influenced by the variability in specimen geometry, loading conditions and materials properties $[14,15]$.

The general belief is that the micro-tensile and micro-shear bond tests are the most suitable methods available to evaluate bond strength of dental adhesive. However, there is a lack of published evidence concerning the most accurate methodology when testing bond strength to enamel. Micro-shear bond strength test appears to be more accurate in differentiating among the stronger adhesives (micro-shear is better than micro-tensile) [16]. Mainly because of its overall simplicity, several variations of shear bond testing have been widely used. Among the advantages are ease of specimen preparation, simple test protocol, and the ability, at least qualitatively, to rank different products according to bond strength values [17]. The small size test specimens permits many tests to be performed on the same substrate. Thus, it can provide accurate and reliable shear bond strength data. Shear bond strength testing with bonded cross-sectional areas of $1 \mathrm{~mm}^{2}$ or less is also referred to as micro-shear (SBS) [18].

The null hypotheses of this study was the micro-shear bond strength of dental composite cured with either a LED based LCU or a conventional halogen LCU does not differ significantly. The primary objective of this study was to test the micro-shear bond strength of dental composite cured with either a LED based LCU or a conventional halogen LCU.

\section{Materials and Methods}

Fifteen caries-free freshly extracted human third molar teeth were debrided using a scalpel to remove attached soft tissues, cleaned with pumice and water, and stored in a refrigerator at $4^{\circ} \mathrm{C}$, for up to 3 months in a $1 \%$ Chloramine-T solution ( $\mathrm{pH} 9.3$ ) prior to use in this in vitro study. All the teeth used belonged to subjects aged inbetween 20 and 40 years. Roots were cut and separated just below the cemento-enamel junction. The crown of each tooth was cut parallel to the long axis of the tooth mesio-distally and buccolingually to give four sections using a slowly rotating diamond blade (Isomet, Buehler, Lake Bluff, IL, USA) under a flowing water. Each segment was stored separately in distilled water at $4^{\circ} \mathrm{C}$ for not more than 4 days. A total of 50 sections were obtained. In order to standardize the enamel reduction, $0.5 \mathrm{~mm}$ depth orientation pits were prepared with air-rotor bur and superficial enamel was removed initially using a superfine diamond bur (SF\# 145, Shofu, Inc., Kyoto Japan) on a high speed hand piece followed by grinding with \#600-grit (Resista, OMEGNA Nia Filli DiDa / Italy) under water cooling on a polishing machine (Tegrapol-25, Struers, Ballerup, Denmark) until a flat enamel surface was obtained. Each section was mounted in cold-curing acrylic resin (IMI cryl, Turkey), in polyvinylchloride molds, such that the flattened enamel surface was flush with the upper edge of the mold. Teeth segments were then ultrasonically cleaned in distilled water for $5 \mathrm{~min}$ prior to the bonding procedure to remove any remaining Silicone Carbide dust particles. Finally, the enamel surfaces were dried with oil-free air and inspected under binocular magnification to ensure that the enamel was grossly intact.

The teeth specimens were divided at random into five groups of ten each. Each group was assigned to a curing technique as follows: Three groups were cured with LED LCU for $10 \mathrm{~s}, 20 \mathrm{~s}$, and 40 s. groups 4 and 5 were cured with halogen LCU for $20 \mathrm{~s}$ and $40 \mathrm{~s}$. The enamel was then etched with $37 \%$ phosphoric acid gel (Super Etch, SDI, Australia) for 30 s [19,20]. After etching the enamel surfaces were washed with water for $20 \mathrm{~s}$ and dried with oil-free air until the typical chalk-like appearance of etched enamel was observed $[19,21]$. The bonding procedures were done under ambient laboratory temperature according to the manufacturer's instructions and the specification TR 11405 of the International Organization for Standardization [22]. Thoroughly saturated brush tip was generously applied as one uniform layer of the adhesive (Alpha-dent bonding resin, Dental Technologies, Lincolnwood, USA) onto the prepared enamel surface with disposable brush and left undisturbed for $20 \mathrm{~s}$. The solvent was removed by blowing gently with air (with the tip of the air syringe approximately $2 \mathrm{~cm}$ from the surface) for 20 seconds and then light-polymerized for $20 \mathrm{~s}$ using either the halogen LCU or LED LCU at zero distance and vertical direction.

PROLUX 570 (Peng Lime, Enterprise Co., LTD, Taiwan) halogen light-curing unite based on halogen technology and a custom made LED LCU were used in our study. The PROLUX 570 uses a fan cooled halogen bulb with a light intensity of 500 $\mathrm{mW} / \mathrm{cm}^{2}$ and wavelength range of $400-510 \mathrm{~nm}$. The light intensity was measured with Demtron Radiometer (Kerr curing Radiometer, Model 100, CT, Danbury, USA).The LED LCU used in this study was bluephase C5 (Ivoclar, Vivadent, Liechtenstein, Austria). It has wavelength range $430-490 \mathrm{~nm}$ and light output was $500 \mathrm{~mW} / \mathrm{cm}^{2}$. The light intensity of the LED LCU was measured by the light-meter which integrated in the hand piece holder.

Translucent polyvinylchloride micro-tube of $0.8 \mathrm{~mm}$ internal diameter and $1.5 \mathrm{~mm}$ high was mounted on the adhesive-covered enamel surface to restrict the bonding area (Figure-1). The iris was held firmly on the surface using a double-sided tape to prevent the resin from seeping away from the defined area at the base. A resin composite (Valux Plus, 3M ESPE, Dental Products, St. Paul, MN, U.S.A shade A2) was filled into the cylinder and a plastic matrix strip was placed over the resin composite and gently pressed flat and light-cured for the tested curing times using the light-curing units. Because the tube was clear, the resin could be thoroughly cured through the iris.

The shear bond strength was measured by micro-shear testing. Before testing, all samples were checked under an optical microscope with magnification of 30 (Olympus SZX-ILLB100, Olympus Optical, Tokyo, Japan) for bonding defects. The samples showing formation of apparent interfacial gaps, air bubble inclusions or any other defects were excluded from the study 
Figure 1. Tube used for composite resin application.

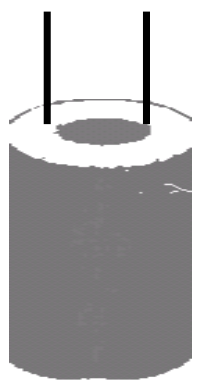

and replaced with new specimens. The samples were subjected to thermal cycling $\left(1500\right.$ cycles) in water baths at $5^{\circ} \mathrm{C}$ and $55^{\circ} \mathrm{C}$ for a dwell time of 30 seconds per bath as recommended by the International Organization for Standardization (ISO TR 11405) $[22,23]$. The embedded enamel specimens were placed in the jig of the universal testing machine (Lloyd instrument LTD, West Fareham, England) such that the enamel surface was flush with the edge of the jig, a thin stainless steel wire loop (diameter 0.2 $\mathrm{mm}$ ) placed around the resin composite cylinder at the enamelcomposite interface, and a load applied at a crosshead speed of $1.0 \mathrm{~mm} / \mathrm{min}$ until bond failure occurred. The maximum load applied $(\mathrm{N})$ at failure for each enamel-composite bond was obtained, converted to $\mathrm{MPa}$ by dividing by the bonded surface area $\left(\mathrm{mm}^{2}\right)$, and recorded as the micro-shear bond strength (MSBS).

After micro-shear bond testing, enamel surfaces were observed under a SEM (JEOL, JSM-5300, Japan). Bond failure was characterized according to the area of resin remaining on the enamel surface and then classified into adhesive, cohesive, or mixed mode of failure. Adhesive mode of failure was recorded if the restorative material was completely detached from the tooth structure (bonded area between enamel, hybrid like enamel layer or overlying adhesive resin). Cohesive failure if the bond failure was entirely within the restorative material or in enamel. Mixed failure if the bond failure was a combination of adhesive and cohesive modes of failure.

\section{Statistical Analysis}

Mean shear bond strengths were analyzed by analysis of variance (One-way ANOVA) and Tukey's test at $\mathrm{P}<0.05$.

\section{Results}

The bond strength data are given in Table-1. One-way ANOVA showed significant differences in microshear bond strengths be- tween curing lights at different times (Table-2, $\mathrm{P} \leq 0.001)$. At 40 $\mathrm{s}$, the halogen VLC produced significantly higher bond strength ratio than that of LED LCU. No significant difference was found between lights at $20 \mathrm{~s}$. There were no significant differences in bond strength ratios between $10 \mathrm{~s}$ and $20 \mathrm{~s}$ LED light and 20 s halogen VLC. At 40 s, the LED LCU produced significantly higher bond strength than that of LED at10 s and $20 \mathrm{~s}$ and halogen VLC at $20 \mathrm{~s}$.

SEM indicated different patterns of bond failures. Most of specimens exhibited cohesive failures after fracture for LED light. However, some specimens of LED showed adhesive failures. Most of specimens exhibited mixed cohesive/adhesive failures after the fracture for halogen VLC. However, some specimens of halogen VLC showed adhesive and cohesive failures. Figure-2 represents the different patterns of failures.

\section{Discussion}

Recently, the micro-shear bond test was introduced as an alternative to the micro-tensile bond test [17] and as a substitute for the conventional shear test. The micro-shear bond test involves the application of a loading force by means of a blade from a universal testing machine to a resin composite cylinder bonded to a substrate disc. The micro-shear bond strength was measured by applying an axial load on the bonded interface using a universal testing machine [24]. The micro-shear bond test method for measuring bond strength was introduced [17]. Compared with the conventional shear bond test, the stress distribution is more concentrated at the interface in the micro-shear bond test [25] which reduces the chance of cohesive failure in the material that does not represent the "true" interfacial bond strength.

Advantages of the micro-shear bond test include less demanding specimen collection and easier control of the bond test area by means of microbore (tygon) tubes [24]. Shimida et al. [26]

Table 1. Mean micro-shear bond strength and standard deviation of composite resin cured with LED and halogen VLC units.

\begin{tabular}{|l|l|l|l|l|l|}
\hline & LED 10 & LED 20 & LED 40 & VLC 20 & VLC 40 \\
\hline Mean & $22.1^{\mathrm{C}}$ & $22.9^{\mathrm{C}}$ & $25.8^{\mathrm{B}}$ & $23.4^{\mathrm{C}}$ & $34.6^{\mathrm{A}}$ \\
\hline SD & 3.3 & 4.1 & 3.4 & 4.3 & 4.5 \\
\hline
\end{tabular}

Table 2. One-way ANOVA results.

\begin{tabular}{|l|l|l|l|l|l|}
\hline & Sum of Squares & df & Mean Square & F & P \\
\hline Between Groups & 1052.122 & 4 & 263.03 & 57.178 & 0 \\
\hline Within Groups & 207.008 & 45 & 4.60 & & \\
\hline Total & 1259.13 & 49 & & & \\
\hline
\end{tabular}


Figure 2. Mode of failure of composite resin: A, Cohesive failure; B, Mixed failure; C, Adhesive failure.

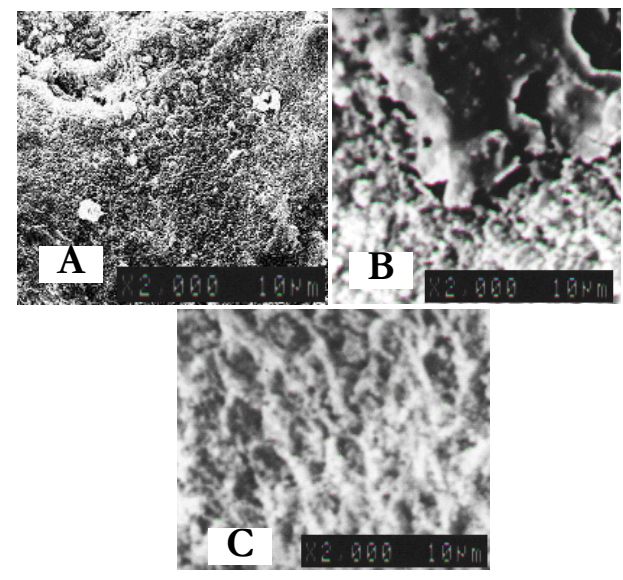

modified the micro-shear bond test by replacing the blade with a looped orthodontic wire. In a study by Foong et al. [24] on bond strength to enamel, the wire micro-shear test was shown to be easier and more reliable compared to the blade micro-tensile test. It can provide accurate and reliable shear bond strength data. The bonding diameters of the specimens have been as small as possible. The smaller diameters give researchers the ability to test several bonded specimens on one flat dentin or enamel surface, thus allowing both for the regional mapping of the mineralized surface and the conservation of extracted teeth needed to provide the necessary substrates. The micro-shear test offers the advantages of facile bond testing for rapid screening of adhesive systems, regional and depth profiling of a variety of substrates for their relative adhesiveness, and conservation of teeth. The micro-shear test also lends itself to in vitro durability studies and may aid in elucidating adhesion mechanisms [17]. Micro-shear bond strength allows for straightforward sample preparation giving precise results preserving the uniformity of the testing area [27].

During the past few years, new generations of lamps- i.e. lightemitting diode (LED) lamps-have been developed. These lamps have irradiance values often in the range of $400-500 \mathrm{~mW} / \mathrm{cm}^{2}$. Despite the lower irradiance compared with halogen lamps, the narrow wavelength range of LED lamps have made them more efficient than the wider wavelength range of halogen lamps. The most efficient wavelength for the degree of polymerization conversion of the resin when camphorquinone is used as initiator has been reported to be $470 \mathrm{~nm}[28,29]$. It has been previously reported that the composite-dentin bond strength decreases as the amount of light energy decreases. Consequently, if insufficient light passes through the composite, then the composite-dentin bond may be reduced [30,31].

Conflicting results are often reported in the literature when the effects of different LCUs on dental restorative materials are studied [7,32]. This may be explained by the differences between irradiation protocols used, especially regarding the intensities. It was concluded that the LED source is more efficient for a comparable overall power output [33]. Rueggeberg et al., [34] observed that the current blue LED visible light curing units are comparable or even superior to high power quartz-tungsten-halogen (QTH) curing devices, due to an enormous increase of power output of high power blue LEDs. The results of the present study indicated that bond strength after curing for 40s with QTH LCU was superior to LED LCU. On the other hand, blue LED LCU was comparable to halogen LCU at 20s. Jandt et al., [35] stated that light activated dental materials will be less well cured with poorer physical properties and an increased risk of premature failure of restora- tions assuming no compensation for decreased LCU irradiance.

Mills et al., [8] indicated that the depths of cure of LED LCU cured fine filled, microfilled, midifilled and hybrid composites of medium shades significantly deeper than did the halogen LCU. Aravamudhan and Rakowskil demonstrated that both light intensity of LED LCU and depth of cure decrease with increasing distance. Fowler et al., [36] concluded that the performance of composites cured with different curing methods has led us to hypothesize that light energy per unit area $\left(\mathrm{J} / \mathrm{cm}^{2}\right)$, rather than light irradiance $\left(\mathrm{mW} / \mathrm{cm}^{2}\right)$, is the critical variable to consider during light curing. With these curing units, dentists were advised to cure 2 -mm thick composite increments for $40 \mathrm{~s}$. The depth of cure of composite resins is mainly dependent on exposure time and distance of the light guide tip of the light source from the composite resin. Furthermore, layer thickness, shade and the translucency of the composite resin have an influence on the depth of cure [2]. Therefore, it can be concluded that the polymerization time can be reduced, if a multiple exposures for composite increments are taken into consideration [37].

Schattenberg et al., [38] concluded that all the blue LED-curing devices cured composite resin sufficiently in an exposure time of $20 \mathrm{~s}$ when a multiple exposure of composite increments was taken into consideration. The post-curing through a second and third layer of resin composite might positively influence the polymerization of the first increment. The minimum exposure time can be reduced to $20 \mathrm{~s}$ or $10 \mathrm{~s}$ with respect to the individual light curing device/resin composite combination.

The manufacturers of LED LCU used in this study stated that $20 \mathrm{~s}$ is valid for light-curing adhesives and a maximum of $30 \mathrm{~s}$ for composite resins with a layer thickness of up to $2 \mathrm{~mm}$. In this study 10 s curing time was suggested to test the ability of reducing the curing time with LED LCU. This study indicted that 10s LED curing time not differs significantly from $20 \mathrm{~s} \mathrm{LED}$ and 20s QTH lights for curing $2 \mathrm{~mm}$ composite resin. So, multiple 10s curing time in incremental application of composite resin was sufficient to get adequate bond strength.

One of the most common methods of testing adhesive systems is the shear bond strength test and has been reported as the most prevalent in literature $[39,40]$. It can provide useful information regarding the bonding mechanism and handling characteristics of adhesives $[39,41]$. Specimens were subjected to thermo-cycling as a means of artificially ageing or weakening bonds prior to testing, as described by the Organization for International Standardization [22]. 
The measured bond between enamel and resin serves two purposes, to help to retain the restoration in place and to help to resist the forces of polymerization shrinkage and hence micro-leakage. It has been estimated that shear bond strength of the order of 17 to $20 \mathrm{MPa}$ is required to achieve these objectives [35,42]. The results obtained from this study fulfills the bond strength requirements when the composite resin was cured at $10 \mathrm{~s}$ LED (22 MPa).

At 40 s curing time, the conventional LCU produced higher bond strength than that of LED LCU at 40s curing time. Both units cured the composite deeper than required by both ISO 4049 . Price et al., [43] concluded that neither of the two LED lights used was able to adequately polymerize the five resin composites tested. The QTH light, which delivered the greatest total energy, always produced the hardest resin composite.

Mavropoulos et al., [44] concluded that a curing time of $10 \mathrm{~s}$ was found to be sufficient to bond metallic brackets to incisors using intensive LED curing units. These new, comparatively inexpensive, curing lamps seem to be an advantageous alternative to conventional halogen lamps for bonding orthodontic brackets.

Higher shear bond strength is equated with enhanced performance, and cohesive failures within tooth structure or composite resin are considered superior to failures within the adhesive layer. A mixed failure mode, with regions of cohesive and adhesive failures, may also occur. When shear bond strength values of adhesive bonding systems exceed the reported shear bond strength of tooth structure, the adhesive resin is assumed to no longer be the limiting factor in bonding success [39]. A strong correlation was found between the mean bond strength and the failure mode: the higher the bond strength, the higher the rate of cohesive failure [45].

\section{Conclusions}

The following conclusions were drawn from the results of this study.

1. Sufficient statistical evidence was provided for the rejection of the null hypotheses concerning invariance of bond strength with curing units and irradiation time at $40 \mathrm{~s}$.

2. Micro shear bond strengths of dental composites cured under laboratory conditions with a LED LCU at $10 \mathrm{~s}$ and $20 \mathrm{~s}$ were statistically equivalent to those cured with a conventional halogen LCU at $20 \mathrm{~s}$.

3. Both units provided sufficient output to exceed the minimum requirements in terms of composites' micro-shear bond strength according to ISO 11405, 1994.

4. Although there was a clear statistical difference between the performance of the QTH and LED LCUs, the LED LCUs' performance can be considered clinically satisfactory.

\section{References}

[1]. El naga AA, Hafez A, El-Shenawy H, Elewa M (2012) Influence of light Curing Mechanism on Microshear Bond Strength of Different Adhesives. J. Amer Sci 8(8):882-887.

[2]. Aravamudhan K, Rakowski, D (2006) Variation of depth of cure and intensity with distance using LED curing lights. Dent. Mater 22(11):988-994.

[3]. Fan PL, Schumacher RM, Azzolin K, Geary R, Eichmiller FC ( 2002)
Curing-light intensity and depth of cure of resin-based composites tested according to international standards. J. Am. Dent. Assoc 133(4):429-434.

[4]. Barghi N, Berry T, Hatton C (1994) Evaluating intensity output of curing lights in private dental offices. J. Am. Dent. Assoc 125(7):992-996.

[5]. 5.Rueggeberg FA, Twiggs SW, Caughman WF, Khajotia S (1996) Lifetime intensity profiles of 11 light-curing units. J. Dent. Res 75:380.

[6]. Gonçalves BRA, de Moraes RR, Costa AR, Correr AB, Nouer PRA, et al (2011) Effect of Etching Time and Light Source on the Bond Strength of Metallic Brackets to Ceramic. Braz. Dent. J 22(3):245-248.

[7]. Banerjee S, Banerjee R (2011) A comparative evaluation of the shear bond strength of five different orthodontic bonding agents polymerized using halogen and light-emitting diode curing lights: an in vitro investigation. Indian J. Dent. Res 22(5):731-732.

[8]. Mills RW, Jandt KD, Ashworth SH (1999) Dental composite depth of cure with halogen and blue light emitting diode (LED) technology. Br. Dent. J 186(8):388-391.

[9]. Haitz RH, Craford MG, Weissman RH (1995) Handbook of optics (2nd edtn) McGraw Hill, New York. 121-123.

[10]. Shortall AC, Harrington E, Wilson HJ (1995) Light curing unit effectiveness assessed by dental radiometers. J. Dent 23(4):227-232.

[11]. Bennett AW , Watts DC (2004) Performance of two blue light-emittingdiode dental light curing units with distance and irradiation-time.Dent $\mathrm{Ma}$ ter 20(1):72-79.

[12]. International Organization for Standardization. ISO10650 :( 2004) Dentistry-powered polymerization activators. Geneva, Switzerland: International Organization for Standardization.

[13]. Dunn WJ, Bush AC (2002) A comparison of polymerization by lightemitting diode and halogen-based light-curing units. J. Am. Dent. Assoc 133(3):335-341

[14]. Ferrari M, Goracci C, Sadek F, Eduardo P, Cardoso C (2002) Microtensile bond strength tests: scanning electron microscopy evaluation of sample integrity before testing. Eur. J. Oral Sci 110(5):385-391.

[15]. Placido E, Meira JB, Lima RG, Muench A, de Souza RM, et al (2007) Shear versus micro-shear bond strength test: a finite element stress analysis. Dent. Mater 23(9):1086-1092.

[16]. El Zohairy AA, Saber MH, Abdalla AI, Feilzer AJ (2010) Efficacy of microtensile versus microshear bond testing for evaluation of bond strength of dental adhesive systems to enamel. Dent. Mater 26(9):848-854.

[17]. McDonough WG, Antonucci JM, He J, Shimada Y, Chiang MY, et al (2002) A microshear test to measure bond strengths of dentin-polymer interfaces. Biomater 23(17):3603-3608.

[18]. Phrukkanon S , Burrow MF, Tyas M (1998) Effect of crosssectional surface area on bond strengths between resin and dentin. Dent. Mater 14(2), $120-128$.

[19]. Hobsona RS, McCabeb JF, Hoggc SD (2001) Bond strength to surface enamel for different tooth types. Dent. Mater 17(2), 184-189.

[20]. Tavares SW, Consani S, Nouer DF, Magnani MBB, Neto JSP, RomanoFL (2003) Evaluation in vitro of the shear bond strenght of aluminum oxide recycled brackets. Braz. J. Oral Sci 2(7):378-381.

[21]. Padipatvuthikul P, Mair LH (2008) Comparison of Shear Bond Strength, Fatigue Limit and Fatigue Life in resin-bonded metal to enamel bonds. Dent. Mater 24(5):674-680.

[22]. International Organization for Standardization (1994) - Guidance on testing of adhesion to tooth structure. ISO/TC106/SC 1 N236, Resolution 6 1. - CD. TR. 11405, trieste, October.

[23]. Bourke BM, Rock WP (1999) Factors Affecting the Shear Bond Strength of Orthodontic Brackets to Porcelain. J. Orthodont 26(4): 285-290.

[24]. Foong J, Lee K, Nguyen C, Tang G, Austin D et al (2006) Comparison of microshear bond strengths of four self-etching bonding systems to enamel using two test methods. Aust. Dent. J 51(3):252-257.

[25]. Banomyong D, Palamara J, Burrow MF, Messer HH (2007) Effect of dentin conditioning on dentin permeability and microshear bond strength. Eur. J. Oral. Sci 115(6): 502-509.

[26]. Shimada Y, Kikushima D, Tagami J (2002) Micro-shear bond strength of resin-bonding systems to cervical enamel. Am. J. Dent 15(6):373-377.

[27]. Senawongse P, Sattabanasuk V, Shimada Y, Otsuki M, Tagami J (2004) Bond strength of current adhesive systems on intact and ground enamel. J. Esthet. Restor. Dent 16(2):107-115.

[28]. Emami N, Söderholm KJM (2003) How light irradiance and curing time affect monomer conversion in light-cured resin composites. Eur. J. Oral Sci 111(6):536-542.

[29]. Nomoto R (1997) Effect of light wavelength on polymerization of light cured resins. Dent. Mater 16(1):60-73.

[30]. Hinoura K, Miyazaki M, Onose H (1991) Effect of irradiation time to lightcured resin composite on dentin bond strength. Am. J. Dent 4(6):273-276.

[31]. Miyazaki M, Hinoura K, Onose H, Moore BK (1995) Influence of light intensity on shear bond strength to dentin. Am. J. Dent 8(5):245-248.

[32]. D'Alpino PH, Wang L, Rueggeberg FA, Svizero NR, Pereira JC et al (2006) Bond strength of resin-based restorations polymerized with different light- 
curing sources. J. Adhes. Dent 8(5):293-298.

[33]. Oberholzera TG, Du Preeza IC, Kidd M (2005) Effect of LED curing on the microleakage, shear bond strength and surface hardness of a resin-based composite restoration. Biomater 26(18):3981-3986.

[34]. Rueggeberg FA, Blalock JS, Callan RS (2005) LED curing lights-what's new? Compend. Contin. Educ. Dent 26(8):586-591.

[35]. Jandt KD, Mills RW, Blackwell GB, Ashworth SH (2000) Depth of cure and compressive strength of dental composites cured with blue light emitting diodes (LEDs). Dent. Mater 16(1):41-47.

[36]. Fowler CS, Swartz ML, Moore BK (1994) Efficacy testing of visible-lightcuring units. Oper. Dent 19(2):47- 52.

[37]. Vandewalle KS, Roberts HW, Andrus JL, Dunn WJ (2005) Effect of light dispersion of LED curing lights on resin composite polymerization. J. Esthet. Restor. Dent 17(4):244-254.

[38]. Schattenberg A, Lichtenberg D, Stender E, Willershausen B, Ernst CB (2008) Minimal exposure time of different LED-curing devices. Dent. Mater 24(8):1043-1049.

[39]. 0flo G (1993) Bond strength testing: what does it mean? Int. Dent. J
43(5):492-498.

[40]. Özcan M , Vallittu PK (2003) Effect of surface conditioning methods on the bond strength of luting cement to ceramics. Dent. Mater 19(8):725-731.

[41]. Perdigao J, Swift Jr , Cloe BC (1993) Effects of etchants, surface moisture, and resin composite on dentin bond strengths. Am. J. Dent 6(2):61-64.

[42]. Davidson C, deGee A, Fielzer A (1984) The competition between the composite-dentin bond strength and the polymerization contraction stresses. J. Dent. Res 63(12):1396-1399.

[43]. Price RB, Ehrnford L, Andreou P, Felix CA (2003) Comparison of quartzhalogen, light-emitting diods and plasma arc curing lights. J. Adhes. Dent 31(3):193-207.

[44]. Mavropoulos A, Staudt C , Kiliaridis S, Krejci I (2005) Light curing time reduction: in vitro evaluation of new intensive light-emitting diode curing units. Eur. J. Orthodont 27(4):408-412.

[45]. Van Meerbeek B, Peumans M, Poitevin A, Mine A, Van Ende A, et al (2010) Relationship between bond-strength tests and clinical outcomes. Dent. Mater 26(2):100-121. 